OPEN ACCESS

Edited by:

Alessandro Antonelli,

University of Pisa, Italy

Reviewed by:

Aime Franco,

Children's Hospital of Philadelphia,

United States

Hideyuki Iwayama,

Aichi Medical University, Japan

${ }^{*}$ Correspondence:

Zhihui Li

zhihuiliwt@163.com

Specialty section: This article was submitted to

Thyroid Endocrinology,

a section of the journal

Frontiers in Endocrinology

Received: 07 July 2021 Accepted: 17 September 2021

Published: 05 November 2021

Citation:

Wang Y, Zhou S, Wang D, Wei T, Zhu J and Li Z (2021) Complement C4-A and Plasminogen as Potential Biomarkers for Prediction of Papillary

Thyroid Carcinoma.

Front. Endocrinol. 12:737638.

doi: 10.3389/fendo.2021.737638

\section{Complement C4-A and Plasminogen as Potential Biomarkers for Prediction of Papillary Thyroid Carcinoma}

\author{
Yichao Wang ${ }^{1,2}$, Shengliang Zhou ${ }^{1,2,3}$, Dun Wang ${ }^{1,2,3}$, Tao Wei ${ }^{1,2}$, Jingqiang Zhu ${ }^{1,2}$ \\ and Zhihui $\mathrm{Li}^{1 *}$ \\ ${ }^{1}$ Department of Thyroid \& Parathyroid Surgery Center, West China Hospital, Sichuan University, Chengdu, China, \\ 2 Laboratory of Thyroid and Parathyroid Disease, Frontiers Science Center for Disease-related Molecular Network, West \\ China Hospital, Sichuan University, Chengdu, China, ${ }^{3}$ West China School of Medicine, West China Hospital, Sichuan \\ University, Chengdu, China
}

Background: Early diagnosis and therapy of papillary thyroid carcinoma (PTC) is essential for reducing recurrence and improving the long-term survival. In this study, we aimed to investigate the proteome profile of plasma and screen unique proteins which could be used as a biomarker for predicting PTC.

Methods: Serum samples were collected from 29 PTC patients and 29 nodular goiter (NG) patients. Five PTC serum samples and five NG serum samples were selected for proteome profiles by proteomics. Eight proteins in PTC and NG serum samples were selected for confirmation by enzyme-linked immunosorbent assay analysis. Receiver operating characteristic curves was used to evaluate the diagnostic value of potential biomarkers.

Results: Complement C4-A (C4A) and plasminogen (PLG) were significantly lower in serum samples of PTC patients compared with NG patients. C4A was observed to have excellent diagnostic accuracy for PTC, with a sensitivity of $91.67 \%$ and specificity of 83.33\%. The diagnostic value of PLG for PTC was demonstrated by a sensitivity at $87.50 \%$ and specificity at $75.00 \%$. The AUC for C4A and PLG was $0.97 \pm 0.02$ and $0.89 \pm 0.05$.

Conclusion: C4A and PLG appeared to be excellent potential biomarkers for the prediction of PTC.

\section{Keywords: papillary thyroid carcinoma, biomarker, complement C4-A, plasminogen, prediction}

Abbreviations: PTC, papillary thyroid carcinoma; NG, nodular goiter; ELISA, enzyme-linked immunosorbent assay analysis; C4A, complement C4-A; PLG, plasminogen; TNM, tumor node metastasis; VAT1, synaptic vesicle membrane protein VAT-1 homolog (fragment); SERPINA10, protein Z-dependent protease inhibitor; APOB, apolipoprotein B-100; CFI, complement factor I; CD93, complement component C1q receptor; ECM1, extracellular matrix protein 1; ITN, indeterminate thyroid nodules. 


\section{INTRODUCTION}

Thyroid carcinoma is the most common endocrine malignancy with increasing incidences worldwide (1). Epidemiologic studies have shown that thyroid cancer is responsible for 567,233 new cases and 41,071 deaths in 2018 worldwide (2). The disease may rank as the third leading cancer in women (3). Papillary thyroid carcinoma (PTC) comprises more than $80 \%$ of thyroid cancer. Cervical lymph node metastases and aggressive subset are related to the risk of recurrence or death $(4,5)$. The etiology of PTC is not well clarified, and its occurrence is unpredictable. Early and accurate diagnosis of PTC is essential for prevention of progression and recurrence.

Thyroid sonography and fine-needle aspiration biopsy (FNAB) are used for differentiating benign and malignant thyroid lesion. However, FNAB is invasive and may yield $20 \%$ indeterminate cytology (6). Many molecular testing techniques have been made to aid in the diagnosis of PTC, such as ThyroSeq mutational panel, the Afirma gene expression classifier, immunohistochemical stains, and proteomic analysis $(7,8)$. However, these tests are based on biopsy samples and incapable of timely monitoring the occurrence of PTC. Therefore, accurate and timely biomarkers of serum protein are required to predicting PTC. With respect to serum protein for distinguishing PTC, $\mathrm{Hu}$ et al. (9) showed that serum vascular adhesion protein-1 with a $66.7 \%$ sensitivity and $77.4 \%$ specificity was profoundly downregulated in thyroid cancer group which included PTC and follicular thyroid carcinoma. Lu et al. (10) reported that serum complement $\mathrm{C} 4-\mathrm{A} / \mathrm{B}$ increased in PTC patients by using weak cation exchange magnetic beads fractionation followed by matrix-assisted laser desorption/ ionization-time-of-flight mass spectrometry. However, the specificity and sensitivity for diagnosis is not high enough.

We aimed to screen for novel serum proteomic biomarkers for predicting PTC. Through screening assay using proteomics and enzyme-linked immunosorbent assay analysis (ELISA), we successfully identified that serum C4A and plasminogen (PLG) were proteins that met these requirements.

\section{MATERIALS AND METHODS}

\section{Study Population}

A total of 29 PTC patients and 29 nodular goiter (NG) patients were enrolled in this study between September 2018 and July
2019. Twenty-nine PTC patients included 27 patients diagnosed as primary thyroid malignancy and two as suspicious for malignancy according to cytology result of FNAB. Twentynine NG patients with a maximum diameter of larger than 4 $\mathrm{cm}$ estimated by ultrasonography underwent thyroidectomy based on current Chinese guidelines. Pathological diagnosis with PTC or NG was confirmed by the histopathological result of resected specimens. We excluded those patients combined with other cancer types. The clinical characteristics and demographic information were collected from medical records, such as age, gender, tumor node metastasis (TNM) status, and multiplicity of tumor. The baseline characteristics of PTC cases and NG cases are presented in Tables 1, 2. We excluded patients with comorbid conditions such as other forms of cancer and inflammatory diseases. The present study was approved by the ethics committee of the West China Hospital of Sichuan University, and all patients enrolled in the study signed written informed consent.

\section{Sample Collection}

Five-milliliter peripheral venous blood sample was collected prior to any therapeutic intervention by EDTA-containing vacutainer tube. Samples were subjected to centrifugation at $1,600 \mathrm{rpm}$ and $4^{\circ} \mathrm{C}$ for $10 \mathrm{~min}$. The supernatant was aliquoted and stored at $-80^{\circ} \mathrm{C}$ until use.

\section{Screening Assay Using Proteomics}

Five PTC serum samples and five NG serum samples were used to perform the screening assay by using proteomics. The proteomics were performed by Shanghai OE Biotech. Co., Ltd. according to the manufacturer's suggested instruction. Briefly, 10 $\mu \mathrm{l}$ serum sample was added to the resin slurry in the column, then incubated with gentle shaking for $60 \mathrm{~min}$. Column was added to a $2-\mathrm{ml}$ collection tube and centrifuge at $1,000 \times \mathrm{g}$ for 2 min. The filtrate was collected for further processing. Protein concentration was determined using a bicinchonininc acid protein assay (11). The protein was digested into peptides using filter-aided sample preparation. The digested peptides were desalted using C18-Reverse-Phase SPE Column. Liquid chromatography (LC)-data-dependent acquisition (DDA)/dataindependent acquisition (DIA)-mass spectrometry (MS)/MS was performed by a QE mass spectrometer (Thermo, Waltham, MA,

TABLE 1 | Detailed information of characteristics of study samples used in proteomics.

\begin{tabular}{|c|c|c|c|c|c|}
\hline Number & Age (year) & Sex & TNM classification & Multiplicity & Diagnosis \\
\hline 1 & 49 & Female & T2NOMO & No & PTC \\
\hline 2 & 59 & Female & T1aNOMO & No & PTC \\
\hline 3 & 45 & Male & T1bN1aM0 & No & PTC \\
\hline 4 & 33 & Female & T3bN1aM0 & No & PTC \\
\hline 5 & 49 & Female & T1aNOMO & No & PTC \\
\hline 6 & 40 & Female & - & Yes & $N G$ \\
\hline 7 & 40 & Female & - & Yes & $N G$ \\
\hline 8 & 38 & Male & - & Yes & $N G$ \\
\hline 9 & 47 & Female & - & No & NG \\
\hline 10 & 34 & Female & - & Yes & NG \\
\hline
\end{tabular}

PTC, papillary thyroid carcinoma; NG, nodular goiter. 
TABLE 2 | Detailed information of characteristics of study samples used in ELISA.

\begin{tabular}{|c|c|c|c|c|c|}
\hline Number & Age (year) & Sex & TNM classification & Multiplicity & Diagnosis \\
\hline 1 & 40 & Female & T1bN1aM0 & No & PTC \\
\hline 2 & 53 & Male & T1aN1aM0 & No & PTC \\
\hline 3 & 43 & Female & T3bN1bM0 & No & PTC \\
\hline 4 & 28 & Female & T1aN1bM0 & No & PTC \\
\hline 5 & 22 & Female & T3aN1bM0 & Yes & PTC \\
\hline 6 & 39 & Female & T2N1aM0 & Yes & PTC \\
\hline 7 & 52 & Female & T3bN1aM0 & No & PTC \\
\hline 8 & 28 & Female & T1aN1aM0 & Yes & PTC \\
\hline 9 & 28 & Female & T3aN1aM0 & Yes & PTC \\
\hline 10 & 26 & Female & T3bN1aM0 & Yes & PTC \\
\hline 11 & 26 & Female & T2N1aM0 & No & PTC \\
\hline 12 & 38 & Male & T3bN1aM0 & No & PTC \\
\hline 13 & 54 & Male & T1bN1aM0 & Yes & PTC \\
\hline 14 & 27 & Female & T1aNOMO & No & PTC \\
\hline 15 & 53 & Female & T1aNOMO & No & PTC \\
\hline 16 & 30 & Female & T1bN1aM0 & No & PTC \\
\hline 17 & 53 & Female & T1aNOMO & No & PTC \\
\hline 18 & 52 & Male & T3bN1aM0 & No & PTC \\
\hline 19 & 43 & Female & T3bN1aM0 & No & PTC \\
\hline 20 & 30 & Female & T1bN1aM0 & No & PTC \\
\hline 21 & 34 & Female & T2NOMO & No & PTC \\
\hline 22 & 61 & Female & T1aNOMO & No & PTC \\
\hline 23 & 31 & Female & T3N1bM0 & No & PTC \\
\hline 24 & 33 & Female & T1aN1aM0 & No & PTC \\
\hline 25 & 49 & Female & - & Yes & $N G$ \\
\hline 26 & 58 & Male & - & Yes & $N G$ \\
\hline 27 & 58 & Female & - & Yes & $N G$ \\
\hline 28 & 36 & Female & - & Yes & $N G$ \\
\hline 29 & 49 & Female & - & No & $N G$ \\
\hline 30 & 46 & Female & - & No & $N G$ \\
\hline 31 & 61 & Female & - & Yes & $N G$ \\
\hline 32 & 36 & Female & - & No & $N G$ \\
\hline 33 & 47 & Female & - & Yes & $N G$ \\
\hline 34 & 48 & Female & - & Yes & $N G$ \\
\hline 35 & 52 & Female & - & No & $N G$ \\
\hline 36 & 61 & Female & - & Yes & $N G$ \\
\hline 37 & 72 & Female & - & Yes & $N G$ \\
\hline 38 & 61 & Female & - & Yes & $N G$ \\
\hline 39 & 57 & Female & - & No & $N G$ \\
\hline 40 & 85 & Female & - & Yes & $N G$ \\
\hline 41 & 35 & Female & - & Yes & $N G$ \\
\hline 42 & 63 & Female & - & Yes & $N G$ \\
\hline 43 & 45 & Female & - & No & $N G$ \\
\hline 44 & 57 & Female & - & No & $N G$ \\
\hline 45 & 38 & Male & - & Yes & $N G$ \\
\hline 46 & 66 & Female & - & No & $N G$ \\
\hline 47 & 62 & Female & - & Yes & $N G$ \\
\hline 48 & 22 & Female & - & Yes & $N G$ \\
\hline
\end{tabular}

ELISA, enzyme-linked immunosorbent assay; PTC, papillary thyroid carcinoma; NG, nodular goiter.

USA) equipped with an Easyspray source (Thermo, USA). The LC-MS/MS raw data were imported in Maxquant for labeling free quantification analysis. RAW files were imported into Spectronaut pulsar $\mathrm{X}$ to generate the spectral library. The DIA data were analyzed with Spectronaut Pulsar X.

\section{Enzyme-Linked Immunosorbent Assay}

The levels of synaptic vesicle membrane protein VAT-1 homolog (fragment) (VAT1), protein Z-dependent protease inhibitor (SERPINA10), apolipoprotein B-100 (APOB), complement factor I (CFI), complement component $\mathrm{Clq}$ receptor (CD93), C4A, extracellular matrix protein 1 (ECM1), and PLG in serum were detected using commercial kits purchased from RayBiotec, Inc (Norcross, GA, USA), Novus Biologicals (Centennial, CO, USA), and Abbexa Ltd (Cambridge, UK). The assay procedure was conducted by following the manufacturer's protocols. Diluted serum samples were each added into 96-well plate precoated with an antibody. Plates were incubated at $37^{\circ} \mathrm{C}$ or room temperature with gentle shaking. After washing, tetramethylbenzidine (TMB) substrate solution were added into each well followed by incubation for 10-30 $\mathrm{min}$ at room temperature or $37^{\circ} \mathrm{C}$. After terminating the reactions, the absorbance of each well was recorded on a spectrophotometer (Tecan Trading, Mannedorf, Switzerland) at $450 \mathrm{~nm}$. 


\section{Statistical Analysis}

Continuous variables with a normal distribution were expressed as the mean \pm standard deviation (SD). All statistical analyses were assessed by the Student's $t$-test using GraphPad Software Prism 5 (San Diego, CA, USA). A value of $p<0.05$ was considered statistically significant. The sensitivity and specificity of C4A and PLG for diagnosis of PTC was evaluated by receiver operating characteristic (ROC) curve and the area under the curve (AUC). The ROC curves with an AUC $\geq 0.8$ demonstrate good discriminatory power.

\section{RESULTS}

\section{Screening of Serum Samples}

The serum protein spectrum in five PTC and five NG patients were screened using proteomics. The analysis showed that four proteins were upregulated while eight proteins were downregulated in PTC group compared with NG group (Table 3). Among them, we selected eight proteins for further confirmation analysis, including four upregulated and four downregulated with $p$-value $\leq 0.01$.

\section{Selected Protein Levels in PTC and NG Plasma}

The levels of VAT1, SERPINA10, APOB, CFI, CD93, C4A, ECM1, and PLG were analyzed by ELISA in 24 PTC samples and 24 NG samples. We found that VAT1, SERPINA10, APOB, and CFI exhibited no significant differences between the PTC and NG samples (Figures 1A-D). As shown in Figures 1E-H, the expression levels of CD93, C4A, ECM1, and PLG in the PTC group were significantly decreased compared with the NG group $(p<0.05)$.

\section{Diagnostic Value of Serum C4A and PLG for PTC}

Based on the quantitative results, the diagnostic values of serum C4A and PLG for PTC was evaluated by ROC curve. The cutoff values were chosen by considering the maximum sensitivity and specificity for the diagnosis of PTC. As shown in Figure 2 and
Table 4, the AUC for C4A was $0.97 \pm 0.02$. When the cutoff was set at $10,884 \mathrm{ng} / \mathrm{ml}$, the sensitivity of C4A was $91.67 \%$ and the specificity reached $83.33 \%$ (Figure $\mathbf{2 A}$ ). In addition, the AUC for PLG was $0.89 \pm 0.05$. PLG was also observed to have an excellent diagnostic accuracy for PTC with a respective sensitivity and specificity of $87.50 \%$ and $75 \%$ for a cutoff value of $225.2 \mu \mathrm{g} /$ $\mathrm{ml}$ (Figure 2B).

\section{DISCUSSION}

To the best of our knowledge, this is the first study investigating the expression levels of serum C4A and PLG in PTC and NG plasma by proteomics and ELISA. Based on our study, we found C4A and PLG levels were significantly lower in the PTC group compared with the NG group. C4A and PLG were observed to have an excellent diagnostic accuracy for PTC with high sensitivity and specificity. These findings implied that serum C4A and PLG may be potential biomarkers for predicting PTC. It is known that FNAB is frequently used in clinics for evaluating thyroid nodules. Hahn et al. reported FNAB for the prediction thyroid malignancy at presentation with a sensitivity of $89.6 \%$ and specificity of $98.7 \%$, respectively (12). Nonetheless, this method is invasive. In addition, molecular testing of FNA samples, such as RNA test and DNARNA test, is recommended for indeterminate cytology (13), suggesting the molecular testing technique is not a routine recommendation for evaluating thyroid nodule. Livhits et al. reported that RNA test (Afirma genomic sequencing classifier) showed a sensitivity of $100 \%$ and a specificity of $79.6 \%$ for diagnosing indeterminate thyroid nodules (ITN). The sensitivity of DNA-RNA test (ThyroSeq v3 multigene genomic classifier) for diagnosing ITN reached $96.9 \%$ with a specificity of $84.8 \%$ (14). However, the subjects included were thyroid FNAB specimen. This biopsy is an invasive procedure. In the present study, peripheral venous blood sample were examined via proteomics and ELISA analysis. C4A was observed to have excellent diagnostic accuracy for PTC, with a respective sensitivity and specificity of $91.67 \%$ and $83.33 \%$. The sensitivity and specificity of PLG was $87.50 \%$ and $75.00 \%$ for PTC diagnosis, respectively.

$\mathrm{C} 4 \mathrm{~A}$ is one of inflammatory mediators which plays a role in the formation of immune complexes and regulation of binding of

TABLE 3 | Identification information for differentially expressed proteins between PTC and NG.

\begin{tabular}{|c|c|c|c|c|}
\hline Protein name & Gene name & Fold change & $p$-Value & Regulation \\
\hline Synaptic vesicle membrane protein VAT-1 homolog (fragment) (VAT1) & VAT1 & 3.21 & 0.03 & Up \\
\hline Protein Z-dependent protease inhibitor (SERPINA10) & SERPINA10 & 1.67 & 0.01 & Up \\
\hline Apolipoprotein B-100 (APOB) & $A P O B$ & 1.56 & 0.01 & Up \\
\hline Complement factor I (CFI) & $\mathrm{CFI}$ & 1.54 & 0.02 & Up \\
\hline Complement component C1q receptor (CD93) & CD93 & 0.52 & 0.01 & Down \\
\hline Complement C4-A (C4A) & $\mathrm{C} 4 \mathrm{a}$ & 0.55 & 0.01 & Down \\
\hline Extracellular matrix protein $1(\mathrm{ECM} 1)$ & ECM1 & 0.62 & 0.01 & Down \\
\hline Plasminogen (PLG) & PLG & 0.63 & 0.01 & Down \\
\hline Coagulation factor XIII A chain & F13A1 & 0.45 & 0.02 & Down \\
\hline Platelet factor 4 & PF4 & 0.53 & 0.02 & Down \\
\hline Insulin-like growth factor-binding protein complex acid labile subunit & IGFALS & 0.62 & 0.03 & Down \\
\hline Apolipoprotein F & APOF & 0.28 & 0.04 & Down \\
\hline
\end{tabular}

PTC, papillary thyroid carcinoma; NG, nodular goiter. 
A

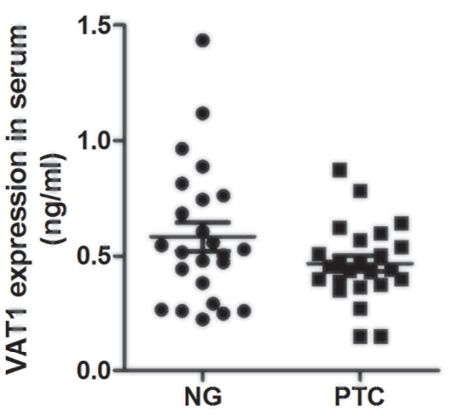

C

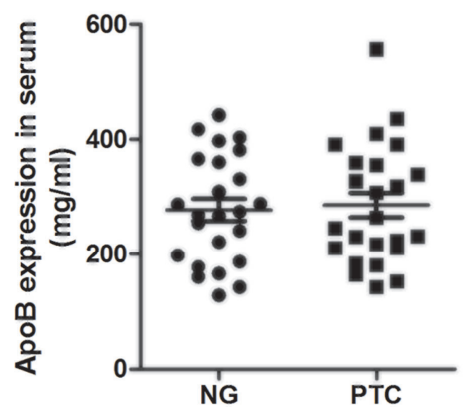

E

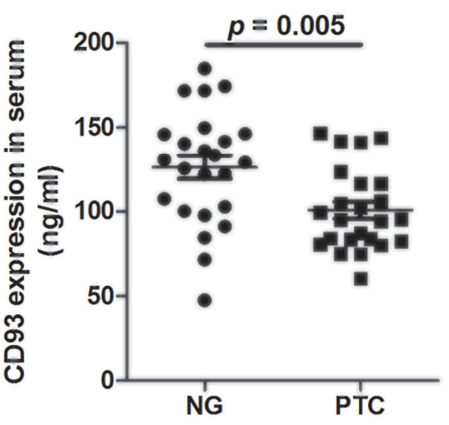

G

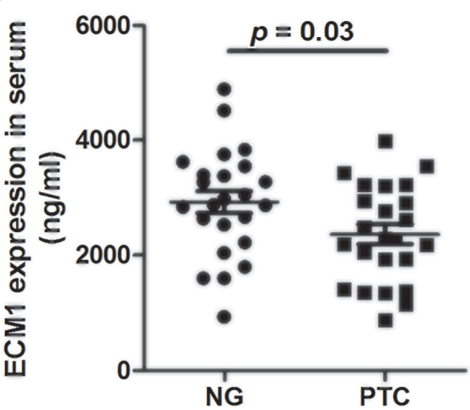

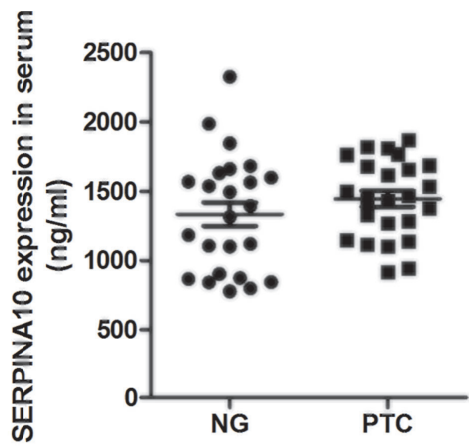

D

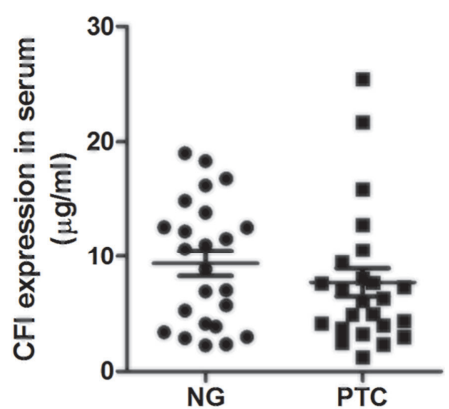

$\mathbf{F}$

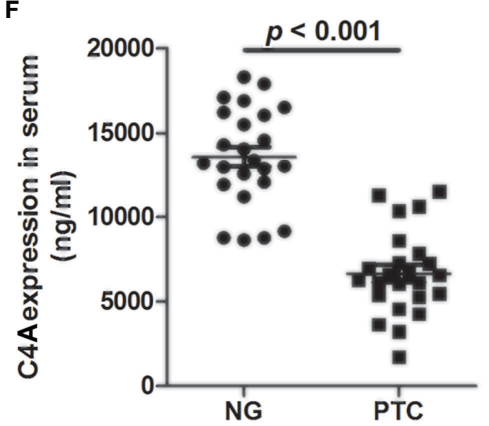

H

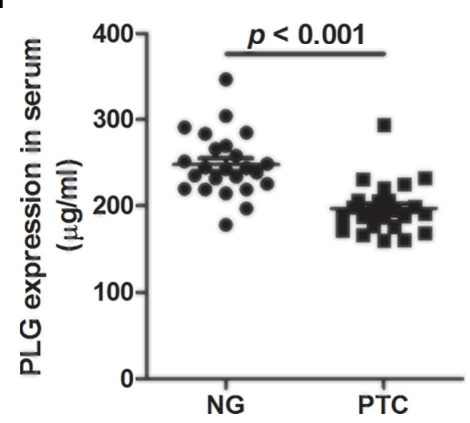

FIGURE 1 | Concentrations of (A) VAT1, (B) SERPINA10, (C) APOB, (D) CFI, (E) CD93, (F) C4A, (G) ECM1, and (H) PLG in the plasma of PTC and NG.

immune aggregates $(15,16)$. In addition, C4 was observed high expression in the thyroid gland compared with the heart, pancreas, and thymus (17). Several studies have surveyed that abnormal C4A expression is associated with some cancers. Tikhonov et al. reported that serum C4A was associated with colorectal cancer developing (16). Zhang et al. reported that the levels of serum C4A in chemosensitive for epithelial ovarian cancer group were significantly higher than in the chemoresistant group (18). Broek 
A

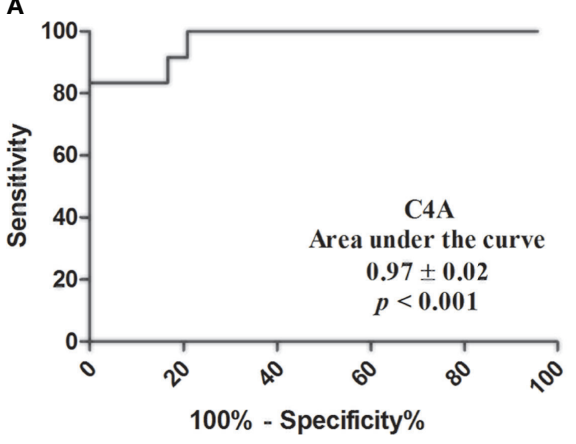

B

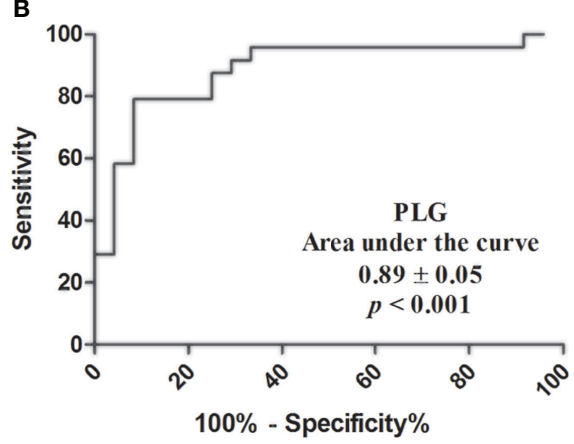

FIGURE 2 | Diagnostic value of C4A and PLG for PTC. (A) Receiver operating characteristic curves of C4A. (B) Receiver operating characteristic curves of PLG.

TABLE 4 | Diagnostic value of C4A and PLG for PTC.

\begin{tabular}{|c|c|c|c|c|}
\hline & Cutoff & Sensitivity $(95 \% \mathrm{Cl})$ & Specificity $(95 \% \mathrm{CI})$ & Likelihood ratio \\
\hline C4A & 10,884 ng/ml & 91.67 (73.00-98.97) & 83.33 (62.62-95.26) & 5.50 \\
\hline PLG & $225.2 \mu \mathrm{g} / \mathrm{ml}$ & $87.50(67.64-97.34)$ & 75.00 (53.29-90.23) & 3.50 \\
\hline
\end{tabular}

C4A, complement C4-A; PLG, plasminogen; PTC, papillary thyroid carcinoma; Cl, confidence interval.

et al. reported that the upregulation of serum C4A was associated with breast cancer (19). Lu et al. identified the upregulation of serum C4A/B in PTC compared with benign thyroid node and healthy individuals (10). In contrast, we found that serum C4A in PTC group was significantly decreased with high sensitivity of $91.67 \%$ and specificity of $83.33 \%$ compared with the NG group. This result might be attributed to the different comparison. However, the mechanism of C4A affecting the tumorigenesis of PTC has not been demonstrated. Further mechanistic analysis is required to understand the biological role of $\mathrm{C} 4 \mathrm{~A}$.

PLG, a 92-94-kilodalton protein produced in the liver and circulated in the blood, is known to be involved in the degradation of extracellular matrix, cell migration, angiogenesis, oncogenesis, and metastasis $(20,21)$. The PLG is associated autophagy of cancer (22). Fang et al. demonstrated that Plasminogen kringle 5 suppressed the growth gastric cancer by inhibiting angiogenesis and apoptosis (23). Yang et al. found that plasminogen kringle 5 can inhibit hepatocellular carcinoma via antiangiogenic activity (24). Tykhomyrov et al. reported that PLGtreated lung adenocarcinoma cells exhibited autophagy induction by upregulation of beclin-1 levels (25). In the present study, we observed that PTC group showed a great downregulation of PLG compared with the NG group, providing insight into the association between PLG and PTC. However, the mechanism by which PLG affects PTC is unknown. Further studies are warranted to exemplify.

Of course, we have to admit several limitations in present study. First, a small number of PTC and NG samples were included in the study. Second, due to financial constraints, we did not investigate serum protein in healthy controls. Third, we have not analyzed how C4A and PLG are involved in tumorigenesis of PTC. Therefore, well-designed trials with larger samples are required to confirm that $\mathrm{C} 4 \mathrm{~A}$ and PLG may be potential biomarkers for the diagnosis of PTC.
In conclusion, this study reveals that serum C4A and PLG may be potentially useful biomarkers for the prediction of PTC. C4A and PLG with a high sensitivity and specificity appear to be developed into a bedside test for rapidly predicting PTC.

\section{DATA AVAILABILITY STATEMENT}

The datasets presented in this study can be found in online repositories. The names of the repository/repositories and accession number(s) can be found below: Proteomexchange [accession: number:PXD029304].

\section{ETHICS STATEMENT}

The studies involving human participants were reviewed and approved by the ethics committee of the West China Hospital of Sichuan University. The patients/participants provided their written informed consent to participate in this study.

\section{AUTHOR CONTRIBUTIONS}

ZL, JZ, and TW designed the research. YW, SZ, and DW performed the experiments and carried out statistical analysis. YW drafted the article. All authors contributed to the article and approved the submitted version.

\section{FUNDING}

This work was supported by Sichuan Science and Technology Program (grant number 2019YJ0038). 


\section{REFERENCES}

1. Siegel RL, Miller KD, Jemal A. Cancer Statistics, 2019. CA Cancer J Clin (2019) 69(1):7-34. doi: 10.3322/caac.21551

2. Bray F, Ferlay J, Soerjomataram I, Siegel RL, Torre LA, Jemal A. Global Cancer Statistics 2018: GLOBOCAN Estimates of Incidence and Mortality Worldwide for 36 Cancers in 185 Countries. CA Cancer J Clin (2018) 68 (6):394-424. doi: 10.3322/caac.21492

3. Aschebrook-Kilfoy B, Schechter RB, Shih YC, Kaplan EL, Chiu BC, Angelos $\mathrm{P}$, et al. The Clinical and Economic Burden of a Sustained Increase in Thyroid Cancer Incidence. Cancer Epidemiol Biomarkers Prev (2013) 22(7):1252-9. doi: 10.1158/1055-9965.EPI-13-0242

4. Podnos YD, Smith D, Wagman LD, Ellenhorn JDI. The Implication of Lymph Node Metastasis on Survival in Patients With Well-Differentiated Thyroid Cancer. Am Surg (2005) 71(9):731-4. doi: 10.1177/000313480507100907

5. Lundgren CI, Hall P, Dickman PW, Zedenius J. Clinically Significant Prognostic Factors for Differentiated Thyroid Carcinoma: A PopulationBased, Nested Case-Control Study. Cancer (2006) 106(3):524-31. doi: $10.1002 / \mathrm{cncr} .21653$

6. Cibas ES, Ali SZ. The 2017 Bethesda System for Reporting Thyroid Cytopathology. Thyroid (2017) 27(11):1341-6. doi: 10.1089/thy.2017.0500

7. Jug RC, Datto MB, Jiang XS. Molecular Testing for Indeterminate Thyroid Nodules: Performance of the Afirma Gene Expression Classifier and ThyroSeq Panel Cancer Cytopathol. Cancer Cytopathol (2018) 126(7):47180. doi: $10.1002 /$ cncy.21993

8. Galli M, Pagni F, De Sio G, Smith A, Chinello C, Stella M, et al. Proteomic Profiles of Thyroid Tumors by Mass Spectrometry-Imaging on Tissue Microarrays. Biochim Biophys Acta Proteins Proteom (2017) 1865(7):81727. doi: 10.1016/j.bbapap.2016.11.020

9. Hu ZG, Zhao PX, Zhang KI, Zang LL, Liao HY, Ma WY. Evaluation of Serum Vascular Adhesion Protein-1 as a Potential Biomarker in Thyroid Cancer. Int J Endocrinol (2016) 2016:6312529. doi: 10.1155/2016/6312529

10. Lu ZL, Chen YJ, Jing XY, Wang NN, Zhang T, Hu CJ. Detection and Identification of Serum Peptides Biomarker in Papillary Thyroid Cancer. Med Sci Monit (2018) 24:1581-7. doi: 10.12659/MSM.907768

11. Wiśniewski JR, Zougman A, Nagaraj N, Mann M. Universal Sample Preparation Method for Proteome Analysis. Nat Methods (2009) 6(5):35962. doi: $10.1038 /$ nmeth.1322

12. Hahn SY, Shin JH, Oh YL, Park KW, Lim Y. Comparison Between Fine Needle Aspiration and Core Needle Biopsy for the Diagnosis of Thyroid Nodules: Effective Indications According to US Findings. Sci Rep (2020) 10 (1):4969. doi: 10.1038/s41598-020-60872-z

13. Haugen BR, Alexander EK, Bible KC, Doherty GM, Mandel SJ, Nikiforov YE, et al. 2015 American Thyroid Association Management Guidelines for Adult Patients With Thyroid Nodules and Differentiated Thyroid Cancer: The American Thyroid Association Guidelines Task Force on Thyroid Nodules and Differentiated Thyroid Cancer. Thyroid (2016) 26(1):1-133. doi: 10.1089/ thy. 2015.0020

14. Livhits MJ, Zhu CY, Kuo EJ, Nguyen DT, Kim J, Tseng CH, et al. Effectiveness of Molecular Testing Techniques for Diagnosis of Indeterminate Thyroid Nodules: A Randomized Clinical Trial. JAMA Oncol (2021) 7(1):70-7. doi: 10.1001/jamaoncol.2020.5935

15. Fredslund F, Jenner L, Husted LB, Nyborg J, Andrersen GR, Sottrup-Jensen L L. The Structure of Bovine Complement Component 3 Reveals the Basis for Thioester Function. J Mol Biol (2006) 361(1):115-27. doi: 10.1016/ j.jmb.2006.06.009
16. Tikhonov D, Kulikova L, Kopylov A, Malsagova K, Stepanov A, Rudnev V, et al. Super Secondary Structures of Proteins With Post-Translational Modifications in Colon Cancer. Molecules (2020) 25(14):3144. doi: 10.3390/ molecules25143144

17. Blanchong CA, Chung EK, Rupert KL, Yang Y, Yang Z, Zhou B, et al. Genetic, Structural and Functional Diversities of Human Complement Components $\mathrm{C} 4 \mathrm{~A}$ and $\mathrm{C} 4 \mathrm{~B}$ and Their Mouse Homologues, Slp and C4. Int Immunopharmacol (2001) 1(3):365-92. doi: 10.1016/S1567-5769(01)00019-4

18. Zhang ZM, Qin KY, Zhang W, Yang B, Zhao C, Zhang X, et al. Postoperative Recurrence of Epithelial Ovarian Cancer Patients and Chemoresistance Related Protein Analyses. J Ovarian Res (2019) 12(1):29. doi: 10.1186/ s13048-019-0499-z

19. Broek IVD, Sparidans RW, Schellens JHM, Beijnen JH. Quantitative Assay for Six Potential Breast Cancer Biomarker Peptides in Human Serum by Liquid Chromatography Coupled to Tandem Mass Spectrometry. J Chromatogr B Analyt Technol BioMed Life Sci (2010) 878(5-6):590-602. doi: 10.1016/ j.jchromb.2010.01.011

20. Law RH RH, Abu-Ssaydeh D, Whisstock JC. New Insights Into the Structure and Function of the Plasminogen/Plasmin System. Curr Opin Struct Biol (2013) 23(6):836-41. doi: 10.1016/j.sbi.2013.10.006

21. Bharadwaj AG, Holloway RW, Miller VA, Waisman DM. Plasmin and Plasminogen System in the Tumor Microenvironment: Implications for Cancer Diagnosis, Prognosis, and Therapy. Cancers (Basel) (2021) 13 (8):1838. doi: $10.3390 /$ cancers 13081838

22. Heissig B, Salama Y, Osada T, Okumura K, Hattori K. The Multifaceted Role of Plasminogen in Cancer. Int J Mol Sci (2021) 22(5):2304. doi: 10.3390/ ijms22052304

23. Fang S, Hong H, Li L, He D, Xu Z, Zuo S, et al. Plasminogen Kringle 5 Suppresses Gastric Cancer via Regulating HIF-1 $\alpha$ and GRP78. Cell Death Dis (2017) 8(10):e3144. doi: 10.1038/cddis.2017.528

24. Yang X, Cheng R, Li C, Cai W, Ma JX, Liu Q, et al. Kringle 5 of Human Plasminogen Suppresses Hepatocellular Carcinoma Growth Both in Grafted and Xenografted Mice by Anti-Angiogenic Activity. Cancer Biol Ther (2006) 5 (4):399-405. doi: 10.4161/cbt.5.4.2511

25. Tykhomyrov AA, Nedzvetsky VS, A ĝca CA, Guzyk MM, Korsa VV, Grinenko TV. Plasminogen/plasmin Affects Expression of Glycolysis Regulator TIGAR and Induces Autophagy in Lung Adenocarcinoma A549 Cells. Exp Oncol (2020) 42(4):270-6. doi: 10.32471/exp-oncology.2312-8852.vol-42-no4.15253

Conflict of Interest: The authors declare that the research was conducted in the absence of any commercial or financial relationships that could be construed as a potential conflict of interest.

Publisher's Note: All claims expressed in this article are solely those of the authors and do not necessarily represent those of their affiliated organizations, or those of the publisher, the editors and the reviewers. Any product that may be evaluated in this article, or claim that may be made by its manufacturer, is not guaranteed or endorsed by the publisher.

Copyright (c) 2021 Wang, Zhou, Wang, Wei, Zhu and Li. This is an open-access article distributed under the terms of the Creative Commons Attribution License (CC BY). The use, distribution or reproduction in other forums is permitted, provided the original author(s) and the copyright owner(s) are credited and that the original publication in this journal is cited, in accordance with accepted academic practice. No use, distribution or reproduction is permitted which does not comply with these terms. 